\title{
Occult Primary Thyroid Carcinoma Presenting as Lateral Cervical Mass: Report of Two Cases
}

\author{
Shalini Bahadur, Priyanka Anand*, Kamlesh Prajapati and Namrata Nargotra \\ Dept. of Pathology; NDMC and Hindu Rao hospital. New Delhi, India
}

\begin{abstract}
Background: Thyroid cancer presenting with cervical lymphadenopathy as a sole occurrence is uncommon. It usually presents as a palpable thyroid mass or nodule. Isolated cervical lymphadenopathy is rare and hence poses a diagnostic challenge. Fine needle aspiration- cytology (FNAC) or biopsy are important modalities which help establish the origin.

Case Report: Two cases of lateral cervical lymphadenopathy with no other specific clinical findings were encountered within a span of two weeks with different clinical backgrounds. First to present was a 28 year old young male with a months' history of lateral cervical swelling. Later a 58 year old male also presented without any history of overt primary thyroid carcinoma. FNAC in both the cases confirmed presence of metastasis from occult primary papillary cancer thyroid. Following this ultrasonography neck was performed which revealed a solid-cystic mass in both the cases. In the younger male an ill-defined solid nodule with microcacifications was identified in the left thyroid lobe while in the case of elderly male; thyroid gland was normal. Subsequently both underwent total thyroidectomy with left neck dissection and histologically proven primary papillary thyroid cancer was found.
\end{abstract}

Conclusion: Posterior cervical lymphadenopathy occurring primarily as a result of papillary carcinoma thyroid is rare.

Keywords: Cervical Lymphadenopathy, Papillary Thyroid Cancer, Fine Needle Aspiration- Cytology, Histologic Examination.

\section{Introduction}

Thyroid cancer presenting with cervical lymphadenopathy as sole occurrence is uncommon..$^{[1,2,3]}$ Cervical lymphadenopathy is attributable to a variety of nonneoplastic and neoplastic causes with likelihood of a benign process in younger patients. Metastatic cervical lymphnodes potentially have primary source in head and neck as well as elsewhere and need investigations to guide further management.

Fine needle aspiration cytology (FNAC) or biopsy are important modalities which help establish this origin. We hereby report two cases of papillary carcinoma thyroid presenting as lateral cervical masses.

\section{Case Reports}

Case 1: A 28-year old male patient presented with brief history of a months' duration of an enlarging left neck mass. There were no associated symptoms. On examination a soft to firm non- tender posterior cervical swelling measuring $2 \times 2.5 \mathrm{~cm}$ with restricted mobility was noted. Thyroid gland was not palpable. Baseline investigations including complete blood counts (CBC), electrolytes and thyroid function tests were within normal limits.

A family history of head and neck cancer was denied. Previous exposure to radiation was not found. A FNAC performed showed cells in syncytial aggregates, balls and sheets with anatomical borders. Nuclear crowding and overlapping was noted with relatively abundant cytoplasm. Several of the nuclei showed nuclear grooving as well as occasionally presence of intra- nuclear cytoplasmic inclusions. Possibility of metastatic papillary carcinoma thyroid was suggested on FNAC. Following this Ultrasonography of neck was done.

A $2.5 \mathrm{~cm}$ solid-cystic mass with thick irregular walls was found. An ill-defined hypoechoic solid nodule with micro-calcifications was identified in left thyroid lobe. A Technetium perfusion scan showed evidence of increased perfusion in cold nodule involving left lobe of thyroid gland with homogenous uptake in right thyroid lobe. Thyroidectomy with left neck dissection was performed.

Case 2: A 58-year old male presented with a left sided neck mass of three months' duration. No positive contributory clinical history was noted. On examination a firm, nontender $3 \times 2 \mathrm{~cm}$ mass was noted on left side of neck. FNAC was performed that showed syncytial aggregates and papillary fragments without apparent fibrovascular cores. Nuclei had a powdery chromatin with many showing longitudinal cytoplasmic grooves. Occasional intranuclear cytoplasmic inclusions were evident along with thick streaks of colloid-like material. Again, possibility of metastatic carcinoma thyroid was suggested. USG on follow up revealed a well-defined solid- cystic mass in left side of neck of size $2 \mathrm{cms}$ and both lobes of thyroid showed apparently normal size and echotexture. 


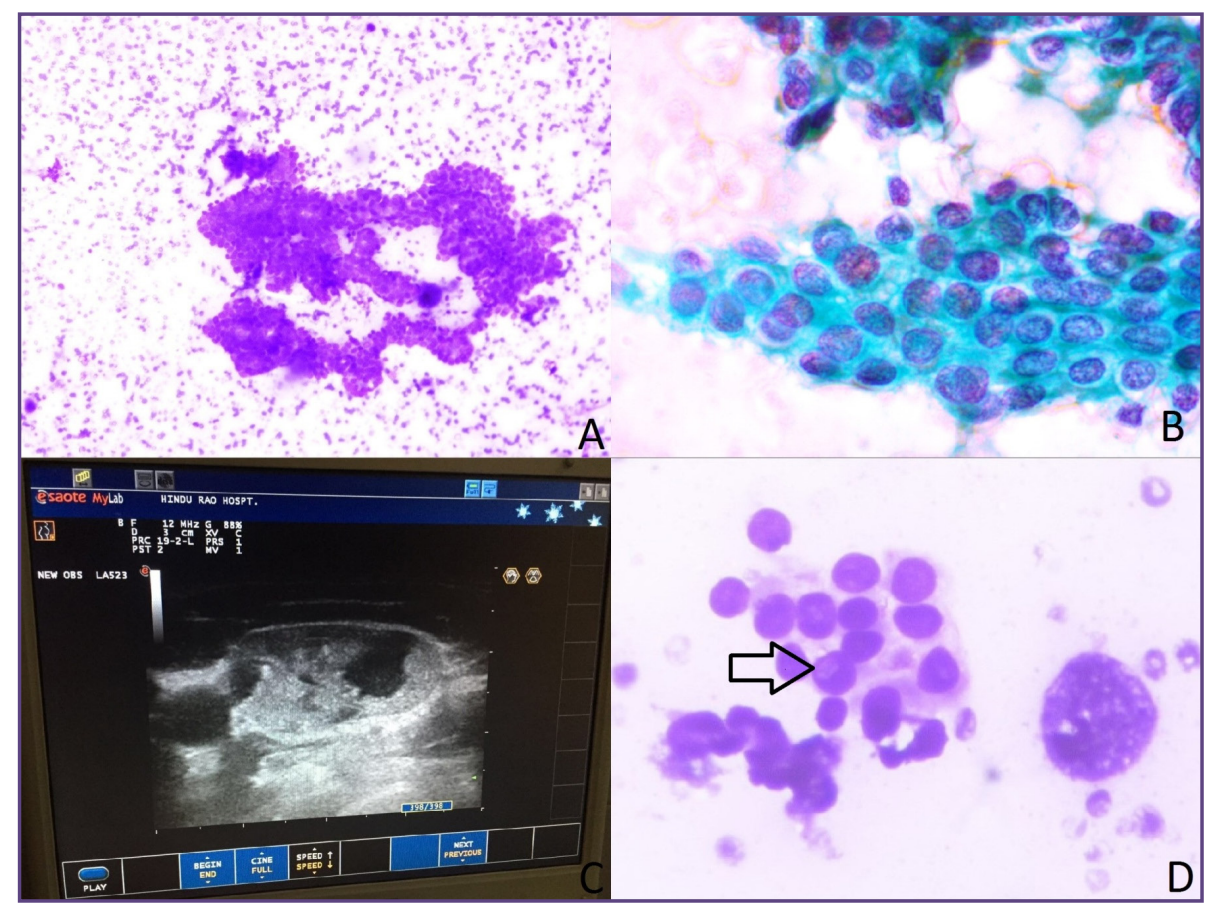

Fig. 1(Case 1): Metastatic Papillary Thyroid Carcinoma in cervical lymph node: (A)- Papillary cytoarchitecture with anatomical edges. (MGG; 10x) (B)-Cells having round to oval nuclei with pale powdery chromatin and many of them showing intranuclear grooves. (Papanicolaou; 100x) (C)- Ultrasonography- A $2.5 \mathrm{~cm}$ solid - cystic mass with thick irregular walls. (D)- Cells with intra-nuclear cytoplasmic inclusion (arrow) with a foamy macrophage seen on the right side of the image. (MGG; 40x).

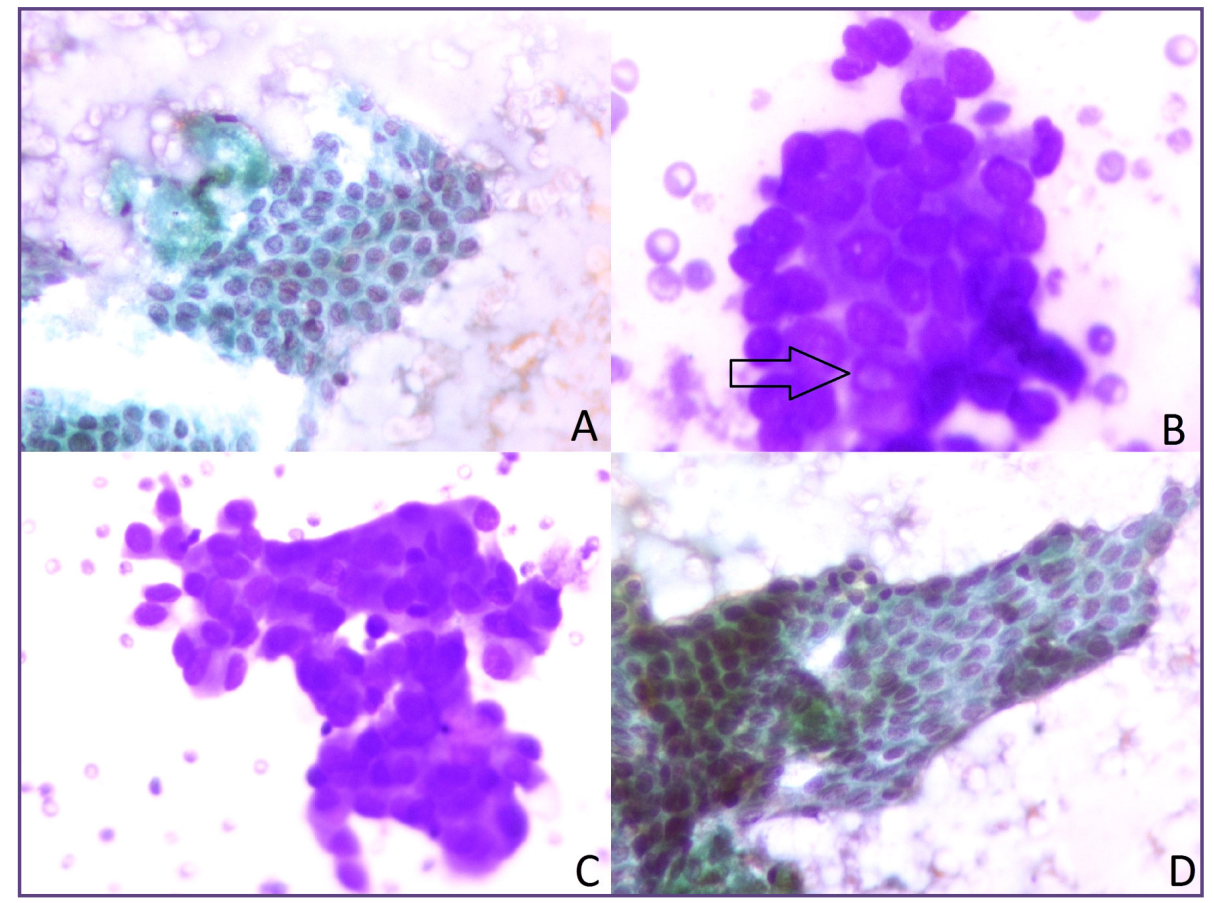

Fig. 2 (Case 2): Metastatic Papillary Thyroid Carcinoma in cervical lymph node: (A)- Cells with intranuclear grooves. (PAP; 40x) (B)- Cells with crowded nuclei and one intra-nuclear cytoplasmic inclusion seen as shown by an arrow. (MGG; 40x) (C)Cells with dense metaplastic squamoid cytoplasm. (MGG; 40x) (D)- Papillae with anatomical borders. (PAP; 10x). 


\section{Discussion}

Thyroid neoplasms commonly present in unsuspecting patients as a thyroid mass. ${ }^{[1,2,4]}$ Less commonly instead of a dominant thyroid lesion, lateral neck mass occurs heralding an occult thyroid primary. Evaluation of a lateral neck mass is diagnostically difficult since a variety of differential diagnosis need to be excluded in both adults and young for which a variety of evaluation options are available. A multitude of inflammatory or congenital lesions need to be considered in younger patients while malignant process considered more likely in elderly. ${ }^{[5]}$

FNAC in a country like India is an easy, simple, uncomplicated and cost-effective diagnostic tool to help clinch diagnosis even when clinicians are least suspecting a malignancy. ${ }^{[6,7,8]}$

Radiological techniques can be a useful adjunct in arriving at a conclusive diagnosis when reviewing cytology smears. Lateral neck mass as an initial manifestation in thyroid cancer has been reported as $10-21 \%{ }^{[1,2,4]}$ but are uncommon particularly to be reported on FNAC alone.

Occult thyroid carcinoma presenting with cervical metastasis has been reported in autopsy studies on histopathology between $10-26 \% \cdot^{[1,2,9-12]}$ Most of these discovered cervical masses have been located in mid jugular (51\%) and low jugular (33\%) lymph nodes followed by supraclavicular $(2 \%)$, posterior cervical $(1.7 \%)$ and high jugular $(10 \%)$ lymph nodes. ${ }^{[2,4]}$ In our cases, posterior cervical lymph nodes were involved with clinically normal thyroid gland without any palpable lumps.

Role of FNAC is very vital in arriving at a presumptive diagnosis with subsequent cyto-histologic co-relation and histologic examination being gold standard. ${ }^{[13]}$

Cytologic evaluation results can be improved by several variables; pathologists expertise and minute observation of cytological details being of utmost importance with equal emphasis on clinical findings.

In present cases, both lateral cervical lymph nodes were cystic. It is imperative to re-assert importance of second aspiration of residual solid masses left after primary fluid aspiration of solid-cystic lumps. This helps to substantially reduce possibility of missing cystic carcinomas. ${ }^{[13]}$ This protocol was strictly adhered to in both cases.

Branchial cleft cysts are common lateral cystic neck masses. Ectopic thyroid tissue within a branchial cleft cyst is a rare phenomenon. Papillary thyroid carcinoma arising from this tissue is an extremely rare possibility and should be kept in mind. ${ }^{[14]}$ Immunohistochemistry helps in the diagnosis of papillary thyroid carcinoma with diffuse cytoplasmic positivity of antibodies against CK19 and CK903 while the benign thyroid follicles stain only weakly and focally for it. ${ }^{[15]}$

Thyroglobulin measurement in FNA material is a useful ancillary test that improves the detection of cystic papillary thyroid cancer metastases. Particulary in cytologically non- diagnostic cases, the measurement of Tg- FNA helps to distinguish benign from malignant cystic lesions. ${ }^{[16]}$ Thyroglobulin levels in $28 \mathrm{yr}$ old patient was borderline and hence inconclusive. It could not be done in the second case.

Multiple criteria of papillary structure without adherent blood vessels, intra-nuclear cytoplasmic inclusions, dense metaplastic cytoplasm, nuclear grooves and thick ropy or blobs of colloid help arrive at correct diagnosis. In cystic degenerations of lymph nodes afflicted by papillary carcinoma metastasis, macrophages have tendency to form cohesive clusters, a feature that if present should raise suspicion of papillary neoplasm and must be sought.

\section{Conclusion}

Posterior cervical lymphadenopathy occurring primarily as a result of papillary carcinoma thyroid is rare. A confident correct diagnosis can be made on cytopathology in combination with clinical evaluation and radiological investigations. This is particularly fruitful in occult nonpalpable lesions that are undetected prior to FNAC. Diagnosis of metastatic papillary carcinoma thyroid warrants follow up by total thyroidectomy with lymph node dissection.

\section{References}

1. Maceri DR, Babyak J, Ossakow SJ. Lateral neck mass. Sole presenting sign of metastatic thyroid cancer. Arch Otolaryngol Head Neck Surg. 1986;112:47-9.

2. Park CS, Min JS. Lateral neck mass as the initial manifestation of thyroid carcinoma. Head Neck. 1989;11:410-13.

3. Machado NO, Chopra PJ, AI Hamdani A. Papillary Carcinoma of the thyroid presenting primarily as cervical lymphadenopathy: An approach to management. Sultan Qaboos Univ Med J. 2009;9(3):328-32.

4. De Jong SA, Demeter JG, Jarosz H, Lawrence AM, Paloyan E. Primary papillary thyroid carcinoma presenting as cervical lymphadenopathy: the operative approach to the lateral aberrant thyroid: Am Surg. 1993;59:172-7.

5. McGuirt FW. Differential diagnosis of Neck Masses. In: CummingC, et al., eds. Otolaryngology Head Neck Surgery. St. Louis: Mosby-Year Book, 1993: 1543-1553.

6. Bagwan IN, Kane SV, Chinoy RF. Cytologic evaluaton of the enlarged neck node: FNAC utility in metastatic neck disease. Int J Pathol 2007;6:2. 
7. Alam K, Khan A, Siddiqui F, Jain A, Haider N, Maheshwari V. Fine needle aspiration cytology (FNAC): A handy tool for metastatic lymphadenopathy. Int J Pathol 2010;10:2.

8. Khajuria R, Goswami KC, Singh K, Dubey VK. Pattern of lymphadenopathy on fine needle aspiration cytology in Jammu. JK Sci 2006;8:157-9.

9. Sanders LE, Rossi RL. Occult well differentiated thyroid carcinoma presenting as cervical node disease. World J Surg 1995;19:642-647.

10. Vassilopoulou-Sellin R, Weber RS. Metastatic cancer as an incidental finding during neck dissection: significance and management. Head Neck 1992;14:459-463.

11. Nussbaum M, Bukachevsky R. Thyroid carcinoma presenting as a regional neck mass. Head Neck Surg 1990;12:114-117.

12. Cady B, Myssiorek D, Thompson N, et al. Management of papillary carcinoma of the thyroid, metastatic to the lateral neck, without apparent primary carcinoma in the thyroid gland. Collected Lett Surg 1993;16:1.

13. Ravetto C, Colombo L, Dottorini ME. Usefulness of fineneedle aspiration in the diagnosis of thyroid carcinoma. Cancer Cytopathol 2000;90:357-363.

14. Mehmood RK, Basha SL, Ghareeb E. A case of papillary carcinoma arising in ectopic thyroid tissue within a branchial cleft cyst with neck node metastasis. Ear, Nose and Throat Journal 2006;85:675-676.

15. Wang Z, Qui S, Mahmoud A. Histopathologic and immunohistochemical characterization of a primary papillary thyroid carcinoma in the lateral cervical lymph node. Experimental and Molecular Pathol 2007;82:91-94.

16. Holmes BJ, Sokoll LJ. Measurement of fine needle aspiration thyroglobulin level increases the detection of metastatic papillary thyroid carcinoma in cystic neck lesions. Cancer Cytopathol 2014;122:521-526.

*Corresponding author:

Dr. Priyanka Anand, Hindu Rao Hospital, NDMC, Malkaganj, New Delhi- 110007. India

Phone: +919990331813

Email: priyankaanand19@yahoo.com

Date of Submission : 07.11.2016

Date of Acceptance : 27.12.2016

Financial or other Competing Interests: None.

Date of Publication : 28.03.2017 